\title{
Sucrose represses the expression of the strigolactone signalling gene D3/RMS4/MAX2 to promote tillering
}

Suyash B. Patil ${ }^{1 \#}$; Francois F. Barbier ${ }^{2 * \#}$; Jinfeng Zhao ${ }^{1}$; Syed Adeel Zafar ${ }^{1}$; Muhammad Uzair ${ }^{1}$; Yinglu Sun ${ }^{1}$; Jingjing Fang ${ }^{1}$; Jessica Bertheloot ${ }^{3}$; Soulaiman Sakr ${ }^{4}$; Franziska Fichtner ${ }^{2}$; Tinashe G. Chabikwa ${ }^{2}$; Shoujiang Yuan ${ }^{5}$; Christine A. Beveridge ${ }^{2 *}$; Xueyong $\mathrm{Li}^{1}{ }^{*}$

${ }^{1}$ National Key Facility for Crop Gene Resources and Genetic Improvement, ICS, CAAS, Beijing 100081, China.

${ }^{2}$ The University of Queensland, School of Biological Sciences, St. Lucia, QLD 4072, Australia.

${ }^{3}$ UMR1345 IRHS, INRAE, SFR 4207 QUASAV, Beaucouzé, France.

${ }^{4}$ UMR1345 IRHS, Agrocampus-Ouest, SFR 4207 QUASAV, Angers, France.

${ }^{5}$ Shandong Rice Research Institute, Jinan 250100, China.

*Correspondence: f.barbier@uq.edu.au; c.beveridge@uq.edu.au; lixueyong@caas.cn \# Authors contributed equally to this work 


\begin{abstract}
:
Shoot branching, which is regulated by a complex signalling network, is a major component of plant architecture and therefore of crop yield. Sugars, acting in a network with hormones, have recently emerged as key players in the control of shoot branching. Previous studies in dicotyledonous plants have shown that sucrose suppresses the inhibitory effect of the plant hormone strigolactone (SL) during this process. The molecular mechanisms underlying this effect are unknown. Here we show that sucrose could antagonise the suppressive action of SL on tillering in rice. At the mechanistic level, we revealed that sucrose alleviates SLmediated degradation of D53. Increase in sucrose availability inhibits the expression of D3, which encodes the orthologue of the arabidopsis F-box MAX2 required for SL signalling. Over-expression of $D 3$ prevented sucrose from inhibiting D53 degradation and enabled the SL inhibition of tillering under high sucrose. The enhanced bud elongation of the $d 3$ mutant to sucrose treatment indicates that suppressed SL perception reduces the minimum amount of sucrose required for sustained bud outgrowth. Decapitation and sugar feeding experiments in pea indicate that RMS4, the D3/MAX2 orthologue in pea, is also involved in the interactions between sucrose and SL. This work shows that D3/MAX2/RMS4 is a key component in the integrating both SL and sugar pathways during the regulation of shoot architecture.
\end{abstract}

Keywords: strigolactones, sugar signalling, shoot branching, plant architecture 


\section{Introduction}

Plants are sessile organisms that have evolved to modify growth and development in order to adapt to constantly changing environmental conditions. Shoot branching regulation allows plants to adjust to a given environment and contributes to the overall plant architecture and is considered an important economic trait for horticulture and agriculture ${ }^{1,2}$. Thus, the study of shoot branching is of major importance for food security given the increasing global population. Shoot branching is regulated

Through a phenomenon called apical dominance, the growing shoot tip maintains a state of dominance over axillary buds. However, factors such as herbivory, pruning, and accidental damage to the main shoot break this dominance, allowing lateral buds to grow into branches. Moreover, depending on the environmental conditions, apical dominance can be reduced, thus allowing plant architecture to adapt to the prevailing conditions. The Shoot tip inhibits axillary bud outgrowth by producing auxin, a phytohormone ${ }^{4-6}$, and by diverting nutrients away from axillary buds due to the strong sink activity generated by its rapid growth $^{7,8}$. Auxin, which is produced by the growing shoot apex and flows downward in the main stem, does not enter the axillary buds ${ }^{9}$. Part of the inhibitory effect of auxin on shoot branching is mediated by the phytohormones strigolactones (SL).

SL are perceived by DWARF14 (D14), an $\alpha / \beta$ hydrolase ${ }^{27,28}$, which then interacts with the Fbox protein DWARF3 (D3), the rice orthologue of the arabidopsis MORE AXILLARY GROWTH2 (MAX2), to form Skp1-Cullin-F-box (SCF) E3 ubiquitin ligase (SCF ${ }^{\mathrm{D} 3 / \mathrm{MAX} 2}$ ) complex ${ }^{29}$. DWARF53 (D53) protein is degraded by the SL-mediated ubiquitination and proteasomal degradation through the $\mathrm{D} 14-\mathrm{SCF}^{\mathrm{D} 3}$ complex $^{30,31}$. The gain-of-function $d 53$ mutants in both rice and arabidopsis display highly branched phenotypes and loss-offunction can rescue the highly branched phenotype of SL deficient and signalling mutants to the wild type (WT) level ${ }^{30,31}$. The TEOSINTE BRANCHED1 (TB1), CYCLOIDEA, and PCF (TCP) family transcription factor TB1 acts as a negative regulator of tillering and is known as an important hub, integrating different signals ${ }^{32,33}$. The D53 protein can directly bind with OsSPL14/IPA1 in rice and can repress its transcriptional activation ${ }^{34}$, while IPA1 has been shown to regulate TB1 expression by directly binding to its promoter ${ }^{35}$. 
Auxin treatment is not always enough to restore the apical dominance ${ }^{16,17}$. As demonstrated upon decapitation in pea, auxin depletion in the stem does not correlate with initial bud growth $^{17,18}$. In this species, this initial bud outgrowth has been correlated with rapid remobilisation of the carbohydrate flow towards the buds and with the accumulation of trehalose 6-phosphate ${ }^{7,8}$, a sugar metabolite mediating sucrose signalling in sink tissues ${ }^{19}$. Reports in other species, and not limited to decapitation assays, also support the involvement of sugars in shoot branching regulation ${ }^{7,20-23}$. A recent study comparing transcriptomes of annual and perennial plants also revealed that bud dormancy was correlated with the expression of genes involved in carbon starvation ${ }^{24}$. In different models, sugar supply to the plant can promote branching ${ }^{8,21,25}$. However, direct sucrose application to monocot plants has still not been reported in the process of shoot branching. The interaction between sugars and hormones during the control of bud outgrowth is not clear. Recent literature highlights that sucrose can antagonise the effect of auxin by inhibiting SL perception to promote bud outgrowth ${ }^{26}$ and that the involvement of cytokinins may be limited to the conditions under which sugars are not readily available ${ }^{15,25,26}$. However, no studies have ever attempted to explore how sucrose and SL interact at the molecular level. Interestingly, sucrose application to single-node cuttings of rose buds can suppress MAX2 and BRANCHED1 (BRC1, the arabidopsis orthologue of TB1) expression ${ }^{20,36}$. In sorghum (Sorghum bicolor), modulation of sugar availability through defoliation and shade treatment affects bud outgrowth and down-regulates MAX2 expression ${ }^{37}$.

The aim of this study is to understand how sugar availability affects the SL-induced tillering inhibition in rice. Using physiology experiments and genetics tools, we sought to identify what component of SL signalling was the target of sucrose during tillering in rice and bud outgrowth in pea. This study will shed light on how environmental factors may regulate branching and tillering at the molecular level, via the control of sugar availability in plants. 


\section{Results}

\section{Sucrose alleviates the effect of SL to promote tillering in rice}

Sucrose has been shown to promote axillary bud outgrowth and alleviate the inhibitory effect of SL during this process in dicotyledonous models ${ }^{21,26}$. However, this antagonistic interaction has not been reported in monocotyledons plants, which have an architecture different compared with eudicots. We therefore tested how sucrose and SL regulate tillering bud outgrowth in rice. To do so, we grew the wild type (WT) Huaidao-5 and the SL-deficient $d w a r f 27$ ( $d 27$ ) tillering mutant hydroponically with different sucrose concentrations with or without $1 \mu \mathrm{M}$ rac-GR24 (a synthetic SL analogue), as concentration that can rescue the tillering phenotype of SL-deficient mutants to WT level ${ }^{27,38}$. We observed that sucrose triggered bud elongation in both WT and SL-deficient $d 27$ plants in a dose-dependent manner with and without GR24 (Figure 1). Interestingly, in the absence of sucrose, tiller bud elongation remained suppressed in the SL-deficient mutant as observed in the WT. With $2 \%$ sucrose, GR24 strongly inhibited tiller bud elongation in both WT (73\%) and the $d 27$ mutant (77\%) (Figure 1c,d). However, with 4\% sucrose, the inhibitory effect of GR24 on tiller bud elongation was reduced (30\% in the WT and $16 \%$ in $d 27$ ). In contrast to sucrose, sorbitol did not promote tillering or alleviate the effect of SL, showing that the effect of sucrose was not due to an osmotic effect (Supplementary Figure S1). Altogether, these results show that sucrose promotes tiller elongation and antagonise SL inhibition of tiller bud outgrowth.

\section{Sucrose inhibits the GR24-induced degradation of D53 protein}

D53 protein and its orthologues in arabidopsis SMXL6, 7 and 8 play a crucial role in SLmediated shoot branching in rice ${ }^{30,31}$ and arabidopsis ${ }^{39,40}$, respectively. Since sucrose reduces SL response, we proposed that sucrose might promote D53 accumulation. We therefore decided to test the impact of sucrose on D53 accumulation. Since dormant tiller buds are very small and D53 protein levels are difficult to detect in shoot tissues, we used rice callus tissues, which have previously been used successfully for this purpose ${ }^{30}$. As predicted, in the absence of GR24 in the growth medium, D53 protein level was strongly increased with the sucrose concentration (Figure 2a). 
We then tested this in presence of GR24. Since the calli grown on different sucrose concentrations accumulate differential D53 levels, calli grown on $4 \%$ sucrose plates were used to start with a similar amount of D53 protein. The calli were washed twice with sterile water to remove exceeding sucrose before being transferred to liquid media containing either $0 \%$ or $4 \%$ sucrose. After 30 minutes of stabilisation, $0.5 \mu \mathrm{M}$ GR24 was supplemented to the $0 \%$ and $4 \%$ sucrose treatments. In the absence of sucrose, D53 protein was degraded within 5 minutes of treatment with GR24. However, in the presence of $4 \%$ sucrose, it took 20 minutes for GR24 to lead to the total degradation the D53 protein (Figure $2 b$ ).

To corroborate this result, we tested OsD53 degradation in response to sucrose and GR24 in tobacco leaves. For this, we fused the OsD53 coding sequence to LUCIFERASE (LUC) and transiently expressed this DNA construct (Supplementary Figure S2) in tobacco (Nicotiana benthamiana) leaves through agroinfiltration. The D53 protein accumulation was assessed by measuring the LUC activity normalised with renilla luciferase values. In the absence of sucrose, the LUC activity was lower in the presence of GR24 than in absence at $4 \mathrm{hr}$ and $8 \mathrm{hr}$ after treatment. However, in the presence of $4 \%$ sucrose, the LUC activity was similar with or without GR24 (Figure 2c). Altogether, these observations show that D53 is higher in the presence of sucrose and that the degradation rate of D53 in response to GR24 is lower in presence of sucrose.

\section{Sucrose inhibits D3/MAX2 expression}

D53 promotes the outgrowth of tillers by inhibiting the expression of the TCP transcription factor gene $T B 1^{32,33}$. Given the accumulation of D53 proteins in response to sucrose (Figure 2), we predicted that sucrose treatment should suppress TB1 expression. As expected, sucrose inhibited the expression of TB1 in rice callus tissues (Figure 3a).

We then determined whether the change of D53 protein levels could be explained by a change in D53 expression at the transcript level. Our results indicate that, in contrast to TB1 expression and D53 protein level, D53 gene expression is not responsive to sucrose (Figure 3b). These observations suggest that sucrose promotes D53 protein accumulation through a post-transcriptional mechanism. 
Given that sucrose reduces SL response and enhances D53 accumulation independent of D53 transcriptional changes, we predicted that sucrose may have an effect on the components of the SCF complex formed by D14 and D3 and which regulate D53 protein degradation ${ }^{29,31} .4 \%$ sucrose significantly reduces the expression of D14 and D3 (2-fold and 3-fold, respectively) (Figure $3 c, d$ ), indicating that sucrose regulation of D14 and D3 expression could be part of the mechanism that enhances D53 accumulation.

We then tested whether the observations made on calli were relevant to shoot base tissues, where tillering occurs. As in calli, sucrose treatment through hydroponic media downregulated the expression of $T B 1$ but not $D 53$ (Figure $3 a, b$ ) in the shoot base. The expression of $D 3$ and $D 14$ genes was also significantly down-regulated by sucrose treatment; this inhibition was much stronger for D3 (56\% and 65\% inhibition with $2 \%$ and $4 \%$ sucrose, respectively) than for $D 14$ ( $16 \%$ and $20 \%$ inhibition with $2 \%$ and $4 \%$ sucrose, respectively) (Figure 3c,d). Both calli and shoot base data support the hypothesis that sucrose suppresses the expression of D14 and D3, two upstream components of D53 degradation.

We then tested whether sucrose could also directly regulate D14 or D3 protein levels. To do so, we tested the impact of $4 \%$ sucrose on D14 and D3 accumulation in calli of transgenic lines over-expressing HA-tagged D14 and D3 under the OsACTIN1 promoter (ACTp:) in the $d 14$ and $d 3$ mutant background, respectively. To confirm this approach, we first showed that the over-expressing lines fully complemented the tiller number and plant height phenotypes of the corresponding $d 14$ and $d 3$ mutants (Supplementary Figure S3). In accordance with previous studies in rice ${ }^{41,42}$, GR24 led to the almost complete degradation D14 protein over $12 \mathrm{~h}$ in the absence of sucrose in the line over-expressing HA-D14. Strikingly, in the presence of sucrose, the GR24-induced D14 degradation was not observed in the same line (Figure 3e). In contrast with D14 protein, neither GR24 nor sucrose showed an effect on D3 protein accumulation in the over-expressing HA-D3 line (Figure 3f). Altogether, these observations show that sucrose does not have a direct inhibitory effect on D14 and D3 protein accumulation, supporting the idea that sucrose preferentially regulates $D 3 / M A X 2$ at the transcriptional level.

\section{D3/MAX2 over expression prevents the sucrose regulation of D53}


If sucrose acts via $D 14$ and/or D3 to regulate D53 accumulation as indicated above (Figure 3), we would predict that over expression of one or both of these two genes would prevent sucrose from promoting D53 accumulation and may prevent sucrose-induced tillering. To test the sucrose response over a short time frame, we grew the calli of ACTp:D3, ACTp:D14 and corresponding WT lines on the NB medium plates with $1 \%$ sucrose. The calli were then washed and rinsed for $60 \mathrm{~min}$ with sterile water. The calli were then shifted to liquid media containing $4 \%$ sucrose and collected after $0 \mathrm{~h}, 2 \mathrm{~h}, 4 \mathrm{~h}$ and $8 \mathrm{~h}$ time points to determine the D53 protein levels. In two WT backgrounds, a steady increase in D53 levels was observed in response to sucrose (Figure 4a,b). D14 over-expression led to higher D53 protein levels in response to sucrose (Figure 4a). Strikingly, the positive effect of sucrose on D53 levels was abolished in the $D 3$ over-expression line (Figure $4 \mathrm{~b}$ ). These results support a hypothesis that $D 3$ regulation, but not $D 14$ regulation, is required for sucrose to enhance accumulation of D53.

If only $D 3$ is necessary for sucrose regulation of the SL response, then $D 3$ over-expression but not $D 14$ over-expression, should prevent sucrose from antagonising the SL-promoted D53 degradation. Accordingly, the effect of GR24 on D53 degradation was delayed by sucrose treatment in the WTs and the ACTp:D14 line, but not in the ACTp:D3 line, in which D53 was totally degraded after 5 minutes of GR24 treatment under high and low sucrose (Figure 4c-f). This result suggests that D3 over-expression prevents sucrose from antagonising the effect of SL on D53 accumulation.

Similar findings to those obtained in rice callus were also observed in tobacco leaves. We tested whether over-expressing $D 3$ in tobacco leaves prevented sucrose from antagonising the effect of SL on D53 accumulation. Indeed, in this system the native tobacco MAX2/D3 expression was also inhibited by sucrose (Figure $4 \mathrm{~g}$ ). We therefore followed the same procedure as described in Figure 2c and co-transfected D53-LUC with a 35S:D3 construct into tobacco leaves. In this system, $4 \%$ sucrose almost totally alleviated the effect of GR24 on D53 degradation when D53-LUC was solely transfected (Figure 4h). However, when the LUC-D53 construct was co-transfected with the 35S:D3 over-expressing construct, sucrose could not prevent the negative effect of GR24 on D53 levels (Figure 4h). These results further support the claim that sucrose alleviates the effect of GR24 on D53 protein levels by inhibiting $D 3 / M A X 2$ expression. 
To further show that the sucrose inhibition of $D 3$ is essential for sucrose to prevent $\mathrm{SL}$ mediated D53 degradation, we grew calli of transgenic lines constitutively expressing D3 and D14 on 4\% sucrose NB plates. As expected, D53 level in the ACTp:D3 line was 65\% lower than in the WT, indicating that D3-over expression prevents the positive effect of sucrose on D53 accumulation (Figure 4i,j). Contrastingly, the D53 protein level was $40 \%$ higher in the ACTp:D14 line than in the WT (Figure 4i,j), consistent again with $D 14$ over-expression failing to suppress the promoting effect of sucrose on D53 protein levels.

\section{D3/MAX2 over-expression prevent the promoting effect of sucrose on tillering}

Given that D53 promotes tillering ${ }^{30,31}$ and that over-expression of $D 3$, but not $D 14$, prevents the sucrose-induced accumulation of D53, we predicted that only D3 over expression would prevent the sucrose-induced tillering. To test this, D3 and D14 over-expressing lines were grown on $0 \%, 0.5 \%, 2 \%$ and $4 \%$ sucrose media with or without $1 \mu \mathrm{M}$ GR2 4 for three weeks and compared with their respective WTs. As recorded in our previous experiment (Figure 1), $4 \%$ sucrose could antagonise the inhibitory effect of GR24 on tiller bud elongation in the WT lines (Figure 5a,c). This effect was also observed in the D14 over-expressing line (Figure 5b). Interestingly, this line was more responsive to sucrose than its corresponding WT, which is in line with the higher D53 protein levels observed in this line (Figure $4 \mathrm{i}, \mathrm{j}$ ). Strikingly, in the D3 over-expressing line, GR24 could still strongly inhibit tiller bud elongation $177 \%$ of inhibition) in the presence of $4 \%$ sucrose (Figure $5 \mathrm{~d}$ ). These results clearly indicate that $D 3$, but not $D 14$ over-expression prevents sucrose from alleviating the inhibitory effect of SL on tillering.

\section{The $d 3$ mutant is hyper-responsive to sucrose}

Our previous results indicate that sucrose and SL interact antagonistically during tillering and that sucrose suppresses the SL pathway through D3/MAX2 inhibition (Figure1,3). Accordingly, knocking out D3/MAX2 may induce hyper-sensitivity to sucrose. We tested this by measuring the response of the loss-of-function $d 3$ and $d 14$ mutants (Supplementary figure S4) to different sucrose concentrations supplied hydroponically (Figure 6). As 
expected, the buds of the $d 3$ mutant responded more than the WT to low sucrose concentrations including $0 \%$ exogenous sucrose (Figure 6a,b). Interestingly, d14 enhanced the growth response to sucrose, but this effect was not always significantly different from the WT and was always lower than $d 3$. Altogether, these data indicate that disrupting SL perception increases responsiveness to sucrose and that $D 3$ disruption has a stronger impact on this phenomenon than $D 14$ disruption, further reinforcing the idea that D3/MAX2 is a major target of sucrose in this process.

\section{PsD3/RMS4/MAX2 is involved in the sucrose-induced bud outgrowth in garden pea}

The role of sugars in bud release has been well described in pea. In this species, decapitation triggers bud outgrowth through redistribution of sugars towards axillary buds, and sucrose feeding can trigger bud release ${ }^{7,8}$. To explore whether a similar mechanism for sucrose regulation of gene expression may occur in pea, we examined the expression of PSD3 and PSD14 in pea as well as their downstream target $B R C 1$, the orthologue of $T B 1^{8,25,26}$ in response to sucrose feeding and decapitation. Similar to the result observed for $D 3$ in rice in response to sucrose, decapitation led to a decrease in PSD3 and BRC1 expression but not in PsD14 (Figure 7a-c). Additionally, sucrose feeding for $4 \mathrm{~h}$ through the petiole strongly inhibited PSD3 expression (70\%) and, to a lesser extent, PSD14 expression (50\%) compared to sorbitol, used as an osmotic control (Figure 7d). BRC1 expression was also repressed by sucrose but not significantly compared with sorbitol at this time point (Figure 7d). The down-regulation of the expression of the sugar-repressible marker gene $D I N 1^{24}$ indicates that the sucrose fed through the petiole reached the bud at this time point (4h). Altogether, these results support the claim that PSD3 is a target for sucrose during bud outgrowth in pea, as observed in rice.

Since PSD3 is also targeted by sucrose in pea, disruption of this gene in pea should lead to hypersensitivity to sucrose, as observed in rice. As predicted, rms4 (mutant in PsD3) buds were hyper-sensitive to sucrose compared with the WT. Again, similar to results in rice, rms3 (mutant in PsD14) was not significantly different from the WT and these mutant buds were substantially shorter under lower sucrose concentration ( $30 \mathrm{mM}$ ) than for rms 4 buds 
(Figure 7e). These results suggest that PsD3/RMS4/MAX2 disruption confers an increased responsiveness of axillary buds to sucrose, similar to results observed in rice (Figure 6).

\section{Discussion}

\section{Sucrose antagonises strigolactone signalling}

Sucrose and SL play a crucial role in shaping plant architecture through their antagonistic action on bud outgrowth, as previously demonstrated in dicotyledonous plants like rose, pea and chrysanthemum ${ }^{20,26,43}$. In the present study, we demonstrated that sucrose also promotes tillering and inhibits the impact of SL on rice tillering. In rice, as in the previously mentioned species, the inhibitory effect of SL on bud outgrowth was almost totally prevented by high sucrose concentrations. The inhibitory effect of sucrose on SL perception is not limited to shoot branching as a recent study showed that sucrose could alleviate the SL action on dark-induced leaf senescence in bamboo leaves ${ }^{44}$.

The expression of the TCP transcription factor $B R C 1$, is well known for its branch inhibition action $^{32,45,46}$, and has previously been reported to be repressed by sucrose in dicot species $^{8,20,47,48}$. Our observations have demonstrated that the expression of $T B 1$, the $B R C 1$ homologue in monocots, is also repressed by sucrose in rice. Furthermore, the D53 protein is up-regulated by sucrose (Figure 2a). Results in rice and tobacco show that the wellestablished SL-mediated D53 degradation was reduced or prevented by sucrose treatment (Figure $2 \mathrm{~b}, \mathrm{c}$ ). This supports the hypothesis that the antagonistic effect of sucrose on the SLmediated bud inhibition ${ }^{8,20,21}$ (Figure 1) is at least partly mediated through sucrose dampening of SL-induced D53 degradation (Figure 8).

\section{Sucrose acts as a transcriptional repressor of $D 3 / M A X 2$}

The F-box protein D3/RMS4/MAX2 plays an essential role in mediating the SL-dependent degradation of D53 protein through the SKP1-CULLIN-F-BOX (SCF) UPS system ${ }^{29,31}$. As observed in other species ${ }^{20,26,49}$, sucrose suppresses $D 3 / M A X 2$ gene expression in rice and pea and this effect is stronger and more consistent than for D14 homologues (Figure 3 a, 
7e). This transcriptional inhibition of $D 3 / M A X 2$ by sucrose in diverse plants suggests that the effect of sucrose on D53 may be mediated at least partly via D3 homologues. This hypothesis is strongly supported by the evidence that sucrose is able to alleviate GR24mediated D53 degradation in a D14 over-expressing line, but not in a D3 over-expressing line. The inability of sucrose to alleviate LUC-D53 protein degradation when co-transfected with the 35S-OsD3 construct provides further support that sucrose targets D3 transcriptional machinery to regulate D53 (Figure $4 \mathrm{i}, 8$ ).

In addition to degradation of D53 proteins, D3 is also responsible for SL-mediated degradation of D14 protein in rice ${ }^{41}$. In light of this, we measured the effect of sucrose on HA-tagged D14 protein levels. In the absence of sucrose, GR24 completely degraded the D14 protein within $12 \mathrm{hr}$, whereas in the presence of $4 \%$ sucrose, GR24 failed to degrade the D14 protein (Figure 3e). This result is important for two reasons. Firstly, it shows that the effect of sucrose on D53 protein levels could not be attributed to a negative effect of sucrose on D14 protein levels. Secondly, it suggests that the effect of sucrose on D53 is likely to be mediated by D3 since the protein accumulation pattern of D14 and D53 reflects what would be expected if D3 was down-regulated ${ }^{41}$ (Figure 8). Contrary to the HA-D14 protein, sucrose did not show any positive or negative effect on HA-D3 protein levels, indicating sucrose regulation through $D 3$ transcription rather than D3 protein stability (Figure 3f).

Interestingly, we observed an over-accumulation of D53 protein in response to sucrose in the D14 over-expressing line. Considering the dual function of D3 protein in modulating both SL-induced D53 and D14 protein degradation, it is possible that there could be a competition effect between D53 and D14 to bind to the D3 protein. Elevated D14 protein levels in the D14 over-expressing line would recruit more D3 protein, creating a deficit for D53 protein degradation. This hypothesis accounts for the over-accumulation of D53 protein levels in the D14 over-expressing line grown at high sucrose concentration (4\%) (Figure $4 \mathrm{i}, \mathrm{j}$ ) as well as the increased tiller bud development observed in the same line (Figure 5).

\section{D3/RMS4/MAX2 acts partly independently of SL to inhibit bud outgrowth}


Our results highlight the fact that rice and pea buds are hyper-responsive to sucrose in the SL perception and signalling mutants $d 14 /$ rms3 and $d 3 /$ max $2 / r m s 4$ (Figure 6,7 ), in accordance with our model (Figure 8). However, we observed that, in both species, responsiveness to sucrose was higher in $d 3 / r m s 4$ than in $d 14 / r m s 3$. This observation suggests that D3/MAX2 may retain an inhibitory effect on bud outgrowth, even in the absence of SL perception. In field conditions, the $d 3$ (s2-215) mutant ${ }^{50}$ showed a more severe dwarf and high tillering phenotype compared with the $d 14$ (htd-2) mutant ${ }^{51}$ both being loss-of-function mutants developed from the same background (Nipponbare) (Supplementary figure S4). This stronger impact of D3/MAX2/RMS4 mutation compared with $D 14 / R M S 3$ mutation or SL-deficiency has been reported in different species and in different developmental processes ${ }^{52-55}$, showing that our observations are not due to a specific allele, and the difference between D3 and D14 mutations is conserved in diverse species. It was previously reported in arabidopsis, that over-expression of MAX2 could partially suppress the decapitation-induced branching in a SL-deficient background ${ }^{56}$, further supporting the claim that D3/MAX2 retains a function independent of SL. Besides mediating SL signalling, D3/MAX2 has been shown to mediate the impact of karrikins in different developmental processes, including seed germination and root development ${ }^{57,58}$. However, karrikins have been reported to have no effect on shoot branching ${ }^{59}$. It is therefore unlikely that the SL-independent effect of D3/MAX2 on branching is dependent on karrikin signalling. Altogether, these observations have demonstrated that D3/MAX2 is an important regulator of bud inhibition and that its inhibition leads to strong responsiveness to sucrose.

\section{Conclusion}

The present study clearly demonstrates that sugar and strigolactone signalling interact during the control of shoot branching, and further demonstrates that sugar availability is an important modulator of this developmental process. SL and sugars are systemic signals and their levels in plants are tightly regulated by environmental cues such as light, moisture and nutrient status of the soil ${ }^{60-65}$. Their interaction during the control of bud outgrowth therefore represents an important regulatory node in the control of plant architecture in 
response to the environment. Additionally, SL and sugars have been shown to be potential tools for crop improvement ${ }^{66,67}$. Our study therefore provides an interesting opportunity for molecular engineering and crop selection to manipulate tillering and ultimately improve crop management and yields. 


\section{Materials and Methods}

\section{Plant material and growth conditions}

For rice, the tiller development assay in response to sucrose and GR24 was performed using HuaiDao5 as wild type and $h d 118^{197 P}$ (d27) as a SL-deficient mutant ${ }^{50}$. The seeds were sterilised as per the method described earlier ${ }^{29}$ with slight modifications. In brief, de-husked seeds were sterilised with $30 \% \mathrm{NaClO}$ solution in a shaker for 30 minutes and then washed with sterilised de-ionised water at least five times. The seeds were directly sown on the solidified $(0.5 \%$ agar) half-strength MS media with different sucrose concentrations with adjusted $\mathrm{pH}$ of 5.8. For the SL treatments, rac-GR24 was used in all the experiments (CX23880, Chiralix). The plants were grown on the different sucrose concentrations for three weeks with or without $1 \mu \mathrm{M}$ GR24 under the $16 \mathrm{hr}$ light $\left(200 \mu \mathrm{mol} . \mathrm{m}^{-2}\right) / 8 \mathrm{hr}$ dark cycle at $28^{\circ} \mathrm{C}$ in a growth chamber. The GR24 and the corresponding treatment combinations were replaced at weekly intervals, maintaining strictly sterile conditions.

The calli of WT (HuaiDao5) grown on NB media plates at $28^{\circ} \mathrm{C}$ in the dark were used for D53 protein degradation assay. The D3 and D14 over-expressing lines and their corresponding mutant and WT lines were used from the earlier work ${ }^{29}$. The lines were maintained in field conditions at the experimental station of Shandong rice research institute, Shandong, China. The lines used for sucrose sensitivity assay consisted of $d 3\left(s 2-215^{0.3935 t o p}\right)$ and $d 14$ (htd-2) mutants in the WT background Nipponbare.

For pea, decapitation and sucrose petiole feeding experiments were performed on the Torsdag L107 background. In vitro sucrose treatment with single nodes was performed as described earlier ${ }^{26}$ in the $r m s 3$, rms 4 mutants and their corresponding wild type Terese. For the decapitation experiment, plants were grown in a glasshouse with a controlled environment ${ }^{7}$. For the sucrose petiole feeding experiment, plants were grown in a growth chamber with 16 hour light $\left(125 \mu \mathrm{mol} / \mathrm{m}^{-2}\right)$ with a temperature of $22^{\circ} \mathrm{C}$ at day and $20^{\circ} \mathrm{C}$ at night.

\section{D53 quantification assay using callus tissues}

The calli were grown on NB media plates containing different sucrose concentrations. Around $250 \mathrm{mg}$ callus tissue was grounded in liquid nitrogen using mortar and pestle to 
make a fine powder. The powder was shifted to $1.5 \mathrm{ml}$ microcentrifuge tubes and mixed with $250 \mu$ l of ice-cold TBT buffer containing (100mM AcOK, 20mM KHEPES pH 7.4, 2mM $\mathrm{MgCl} 2,0.1 \%$ Tween-20,1mM DTT, and $0.1 \%$ protease inhibitor cocktail). The antibody preparation, samples preparation, and protein blots were developed as per the method described earlier ${ }^{30}$. The D53 specific polyclonal antibody produced in mouse was used for immunodetection following 1:1000 dilution in non-fat dairy milk. Anti-HSP-82 or anti-actin was used as a loading control following 1:3000 dilution. Horse-radish peroxidaseconjugated anti-mouse IgG was used as a secondary antibody (CWBIO, Beijing, China) following 1:3000 dilution. The western blots were developed using Tanon ${ }^{\text {TM }}$ High-sig ECL western blotting Substrate (Cat. no:180-5001) with Tanon 6100 chemiluminescent imaging system.

\section{Gene expression analysis}

For rice, shoot base tissues $(0.5 \mathrm{~cm})$ were harvested from two-week-old seedlings grown hydroponically. Total RNA was isolated using RNAprep Pure Plant Kit (Tiangen, Beijing, China, cat. no. DP432) following the manufacturer's instructions. Reverse transcription of 500 ng RNA was performed using the Vazyme, HiScript II Q Select RT SuperMix for qPCR (cat no. R233). Real-time quantitative PCR was performed by Vazymes Cham Q QPCR reagent kit (cat no. Q331) using ABI Prism 7500 Sequence Detection System as per the program recommended by both the instrument and the reagent company. Transcript levels were detected by CT values relative to ACTIN1 as a reference gene. All the primer sequences used in this study are listed in the supplementary table, S1. Gene expression in pea buds was monitored as described earlier ${ }^{68}$.

\section{In vivo luciferase activity assay}

The pCambia1200 vector was modified by integrating Firefly luciferase and Renilla luciferase coding sequences driven by the CaMV35S promoter in addition to the hygromycin resistance marker. The vector then called pCambia1200 35S-LUC had both transient and stable expression capabilities (Figure S2). The coding sequence of the D53 gene was amplified using D53 LUC-F-GGGCGGAAAGGAATTCATGCCCACTCCGGTGG and D53 LUC-RTAGATCCGGTGGATCCTCAACAATCTAGAATTATTCTTGGCGGGAG primer pairs and cloned the Cterminal region of the Firefly luciferase gene using EcoRI and BamHI restriction sites 
following In-fusion ${ }^{\circledR}$ Cloning system by Clonetech, Takara biotech, Japan. The plasmids were transformed into the Agrobacterium strain EHA105 to transfect into tobacco leaves for the transient expression of the D53-Firefly luciferase fused protein. This vector also consists of a Renilla luciferase gene as an internal control which was used to quantify the relative amount of the D53 protein levels. The Agrobacterium strain was transfected into the tobacco leaves as per the methods described earlier ${ }^{69}$. To check the effects of sucrose and GR24 on the transiently expressed D53 levels, different treatment combinations were infiltrated directly into the leaves already transfected with the Agrobacterium strain harbouring luciferase fused D53 construct. The leaves were then harvested in liquid nitrogen, and the activities of Firefly luciferase and Renilla luciferase were determined using the Dual-luciferase ${ }^{\circledR}$ reporter assay system from Promega (cat. no. E1910). The LUC activity was calculated by normalising Firefly luciferase values with Renilla luciferase and was presented as relative luciferase values.

\section{Acknowledgment:}

We would like to thank Catherine Rameau, Alexandre de Saint-Germain and Steven Smith for fruitful discussions about the manuscript. This work was funded as part of a Researcher Development initiative and by the Australian Research Council (Georgina Sweet Laureate Fellowship FL180100139 to Prof. C. A. Beveridge).

\section{References:}

1 Wang, Y. \& Li, J. Molecular basis of plant architecture. Annu Rev Plant Biol. 59, 253-279 (2008).

$2 \mathrm{Li}, \mathrm{H}$. et al. An auxin signaling gene BnaA3.IAA7 contributes to improved plant architecture and yield heterosis in rapeseed. New Phytol. 222, 837-851 (2019).

$3 \quad$ Khush, G. S. Green revolution: the way forward. Nat Rev Genet 2, 815-822 (2001).

$4 \quad$ Cline, M. Concepts and terminology of apical dominance. Am J Bot. 84, 1064 (1997).

5 Thimann, K. V., Skoog, F., William, G. \& Morgan T. H. On the inhibition of bud development and other functions of growth substance in vicia faba. Proc. R. Soc. Lond. B. 114, 317-339 (1934).

6 Thimann, K. V. \& Skoog, F. Studies on the Growth Hormone of Plants: III. The Inhibiting Action of the Growth Substance on Bud Development. Proc Natl Acad Sci U S A 19, 714-716 (1933).

7 Fichtner, F. et al. Trehalose 6-phosphate is involved in triggering axillary bud outgrowth in garden pea (Pisum sativum L.). Plant J 92, 611-623 (2017).

8 Mason, M. G., Ross, J. J., Babst, B. A., Wienclaw, B. N. \& Beveridge, C. A. Sugar demand, not auxin, is the initial regulator of apical dominance. Proc Natl Acad Sci U S A 111, 6092-6097, (2014). 
9 Prasad, T. K. et al. Does Auxin Play a Role in the Release of Apical Dominance by Shoot Inversion in Ipomoea nil? Ann. Bot. 71, 223-229 (1993).

10 Hall, S. M. \& Hillman, J. R. Correlative inhibition of lateral bud growth in Phaseolus vulgaris L. timing of bud growth following decapitation. Planta 123, 137-143 (1975).

11 Morris, D. A. Transport of exogenous auxin in two-branched dwarf pea seedlings (Pisum sativum L.) : Some implications for polarity and apical dominance. Planta 136, 91-96 (1977).

12 Dun, E. A., de Saint Germain, A., Rameau, C. \& Beveridge, C. A. Antagonistic action of strigolactone and cytokinin in bud outgrowth control. Plant Physiol 158, 487-498 (2012).

13 Prusinkiewicz, P. et al. Control of bud activation by an auxin transport switch. Proc Natl Acad Sci U S A 106, 17431-17436 (2009).

14 Sachs, T. The control of the patterned differentiation of vascular tissuesn. Advances in Botanical Research 9, 151-262 (1981)

15 Barbier, F. F., Dun, E. A., Kerr, S. C., Chabikwa, T. G. \& Beveridge, C. A. An update on the signals controlling shoot branching. Trends Plant Sci 24, 220-236 (2019).

16 Beveridge, C. A., Symons, G. M. \& Turnbull, C. G. Auxin inhibition of decapitation-induced branching is dependent on graft-transmissible signals regulated by genes Rms1 and Rms2. Plant Physiol 123, 689-698 (2000).

17 Morris, S. E., Cox, M. C., Ross, J. J., Krisantini, S. \& Beveridge, C. A. Auxin dynamics after decapitation are not correlated with the initial growth of axillary buds. Plant Physiol 138, 1665-1672 (2005).

18 Ferguson, B. J. \& Beveridge, C. A. Roles for auxin, cytokinin, and strigolactone in regulating shoot branching. Plant Physiol 149, 1929-1944 (2009).

19 Figueroa, C. M. \& Lunn, J. E. A Tale of Two Sugars: Trehalose 6-Phosphate and Sucrose. Plant physiol 172, 7-27 (2016).

20 Barbier, F. et al. Sucrose is an early modulator of the key hormonal mechanisms controlling bud outgrowth in Rosa hybrida. J Exp Bot 66, 2569-2582 (2015).

21 Dierck, R. et al. Response to strigolactone treatment in chrysanthemum axillary buds is influenced by auxin transport inhibition and sucrose availability. Acta Physiol.Plant 38, 271 (2016).

22 Evers, J. B. Sugar as a key component of the shoot branching regulation network. Plant, Cell \& Environ. 38, 1455-1456 (2015).

23 Van den Ende, W. Sugars take a central position in plant growth, development and, stress responses. A focus on apical dominance. Front. Plant Sci. 5, 313 (2014).

24 Tarancon, C., Gonzalez-Grandio, E., Oliveros, J. C., Nicolas, M. \& Cubas, P. A Conserved carbon starvation response underlies bud dormancy in woody and herbaceous species. Front. Plant Sci. 8, 788 (2017).

25 Barbier, F. F., Lunn, J. E. \& Beveridge, C. A. Ready, steady, go! A sugar hit starts the race to shoot branching. Curr Opin Plant Biol 25, 39-45 (2015).

26 Bertheloot, J. et al. Sugar suppresses the auxin-induced strigolactone pathway to promote bud outgrowth. New Phytol 225, 866-879 (2019).

27 Arite, T. et al. d14, a strigolactone-insensitive mutant of rice, shows an accelerated outgrowth of tillers. Plant Cell Physiol 50, 1416-1424 (2009).

28 Hamiaux, C. et al. DAD2 is an alpha/beta hydrolase likely to be involved in the perception of the plant branching hormone, strigolactone. Curr Bio/ 22, 2032-2036 (2012).

29 Zhao, J. et al. DWARF3 participates in an SCF complex and associates with DWARF14 to suppress rice shoot branching. Plant Cell Physio/ 55, 1096-1109 (2014).

30 Jiang, L. et al. DWARF 53 acts as a repressor of strigolactone signalling in rice. Nature 504, 401-405 (2013).

31 Zhou, F. et al. D14-SCF(D3)-dependent degradation of D53 regulates strigolactone signalling. Nature 504, 406-410 (2013). 
32 Takeda, T. et al. The OsTB1 gene negatively regulates lateral branching in rice. Plant J 33, 513-520 (2003).

33 Minakuchi, K. et al. FINE CULM1 (FC1) works downstream of strigolactones to inhibit the outgrowth of axillary buds in rice. Plant Cell Physiol 51, 1127-1135 (2010).

34 Song, $X$. et al. IPA1 functions as a downstream transcription factor repressed by D53 in strigolactone signaling in rice. Cell Res 27, 1128-1141 (2017).

35 Lu, Z. et al. Genome-wide binding analysis of the transcription activator ideal plant architecture 1 reveals a complex network regulating rice plant architecture. Plant Cell 25, 3743-3759 (2013).

36 Wang, M. et al. BRANCHED1: A key hub of shoot branching. Front. Plant Sci. 10, 76 (2019).

37 Kebrom, T. H. \& Mullet, J. E. Photosynthetic leaf area modulates tiller bud outgrowth in sorghum. Plant Cell Environ. 38, 1471-1478 (2015).

38 Liu, L. et al. Mutations in the MIT3 gene encoding a caroteniod isomerase lead to increased tiller number in rice. Plant Sci 267, 1-10 (2018).

39 Soundappan, I. et al. SMAX1-LIKE/D53 family members enable distinct MAX2-dependent responses to strigolactones and karrikins in arabidopsis. Plant Cell 27, 3143-3159 (2015).

40 Wang, L. et al. Strigolactone signaling in arabidopsis regulates shoot development by targeting D53-Like SMXL repressor proteins for ubiquitination and degradation. Plant Cell 27, 3128-3142 (2015).

$41 \mathrm{Hu}, \mathrm{Q}$. et al. DWARF14, A receptor covalently linked with the active form of strigolactones, undergoes strigolactone-dependent degradation in rice. Front. Plant Sci. 8, 1935-1935 (2017).

42 Chevalier, F. et al. Strigolactone promotes degradation of DWARF14, an alpha/beta hydrolase essential for strigolactone signaling in Arabidopsis. Plant Cel/ 26, 1134-1150 (2014).

43 Dierck, R. et al. Change in auxin and cytokinin levels coincides with altered expression of branching genes during axillary bud outgrowth in Chrysanthemum. PLoS One 11, (8)e0161732, (2016).

44 Tian, M. Q., Jiang, K., Takahashi, I. \& Li, G. D. Strigolactone-induced senescence of a bamboo leaf in the dark is alleviated by exogenous sugar. J Pestic Sci 43, 173-179 (2018).

45 Aguilar-Martinez, J. A., Poza-Carrion, C. \& Cubas, P. Arabidopsis BRANCHED1 acts as an integrator of branching signals within axillary buds. Plant Cell 19, 458-472 (2007).

46 Braun, N. et al. The pea TCP transcription factor PsBRC1 acts downstream of Strigolactones to control shoot branching. Plant Physiol 158, 225-238 (2012).

47 Gonzalez-Grandio, E. et al. Abscisic acid signaling is controlled by a BRANCHED1/HD-ZIP I cascade in Arabidopsis axillary buds. Proc Natl Acad Sci U S A. 114 (2), E245-E254 (2017).

48 Otori, K., Tamoi, M., Tanabe, N. \& Shigeoka, S. Enhancements in sucrose biosynthesis capacity affect shoot branching in Arabidopsis. Biosci Biotechnol Biochem. 81, 1470-1477 (2017).

49 Kebrom, T. H., Brutnell, T. P., Hays, D. B. \& Finlayson, S. A. Vegetative axillary bud dormancy induced by shade and defoliation signals in the grasses. Plant Signal Behav. 5, 317-319 (2010).

50 Patil, S. et al. An Improved Mesocotyl Elongation Assay for the Rapid Identification and Characterization of Strigolactone-Related Rice Mutants. Agronomy 9, 208 (2019).

51 Liu, W. et al. Identification and characterization of HTD2: a novel gene negatively regulating tiller bud outgrowth in rice. Planta 230, 649-658 (2009).

52 Hayward, A., Stirnberg, P., Beveridge, C. \& Leyser, O. Interactions between auxin and strigolactone in shoot branching control. Plant Physiol 151, 400-412 (2009).

53 Kalliola, M. et al. Differential role of MAX2 and strigolactones in pathogen, ozone, and stomatal responses. Plant Direct 4, 1-14 (2020). 
54 Rasmussen, A. et al. Strigolactones suppress adventitious rooting in Arabidopsis and pea. Plant Physiol 158, 1976-1987 (2012).

55 Umehara, M. et al. Inhibition of shoot branching by new terpenoid plant hormones. Nature 455, 195-200 (2008).

56 Stirnberg, P., Furner, I. J. \& Ottoline Leyser, H. M. MAX2 participates in an SCF complex which acts locally at the node to suppress shoot branching. Plant J 50, 80-94 (2007).

57 Waters, M. T. et al. Specialisation within the DWARF14 protein family confers distinct responses to karrikins and strigolactones in Arabidopsis. Development 139, 1285-1295 (2012).

58 Nelson, D. C. et al. Karrikins Discovered in Smoke Trigger Arabidopsis Seed Germination by a Mechanism Requiring Gibberellic Acid Synthesis and Light. Plant Physiol. 149, 863-873 (2009).

59 Nelson, D. C. et al. F-box protein MAX2 has dual roles in karrikin and strigolactone signaling in Arabidopsis thaliana. Proc Natl Acad Sci U S A. 108, 8897-8902 (2011).

60 Kapulnik, Y. \& Koltai, H. Strigolactone involvement in root development, response to abiotic stress, and interactions with the biotic soil environment. Plant Physiology 166, 560-569 (2014).

61 Yoneyama, K. et al. How do nitrogen and phosphorus deficiencies affect strigolactone production and exudation? Planta 235, 1197-1207 (2012).

62 Yoneyama, K. et al. How do nitrogen and phosphorus deficiencies affect strigolactone production and exudation? Planta 235, 1197-1207 (2012).

63 Yoneyama, K. et al. Nitrogen deficiency as well as phosphorus deficiency in sorghum promotes the production and exudation of 5-deoxystrigol, the host recognition signal for arbuscular mycorrhizal fungi and root parasites. Planta 227, 125-132 (2007).

64 Yoneyama, K., Yoneyama, K., Takeuchi, Y. \& Sekimoto, H. Phosphorus deficiency in red clover promotes exudation of orobanchol, the signal for mycorrhizal symbionts and germination stimulant for root parasites. Planta 225, 1031-1038 (2007).

65 Lemoine, R. et al. Source-to-sink transport of sugar and regulation by environmental factors. Front Plant Sci 4, 272 (2013).

66 Bouwmeester, H. J., Fonne-Pfister, R., Screpanti, C. \& De Mesmaeker, A. Strigolactones: Plant Hormones with Promising Features. Angew. Chem. Int. Ed. 58, 12778-12786 (2019).

67 Fernie, A. R. et al. Synchronization of developmental, molecular and metabolic aspects of source-sink interactions. Nature plants 6, 55-66 (2020).

68 Barbier, F. F. et al. A phenol/chloroform-free method to extract nucleic acids from recalcitrant, woody tropical species for gene expression and sequencing. Plant Methods 15, 62 (2019).

69 Chen, H. et al. Firefly luciferase complementation imaging assay for protein-protein interactions in plants. Plant Physiol 146, 368-376 (2008). 

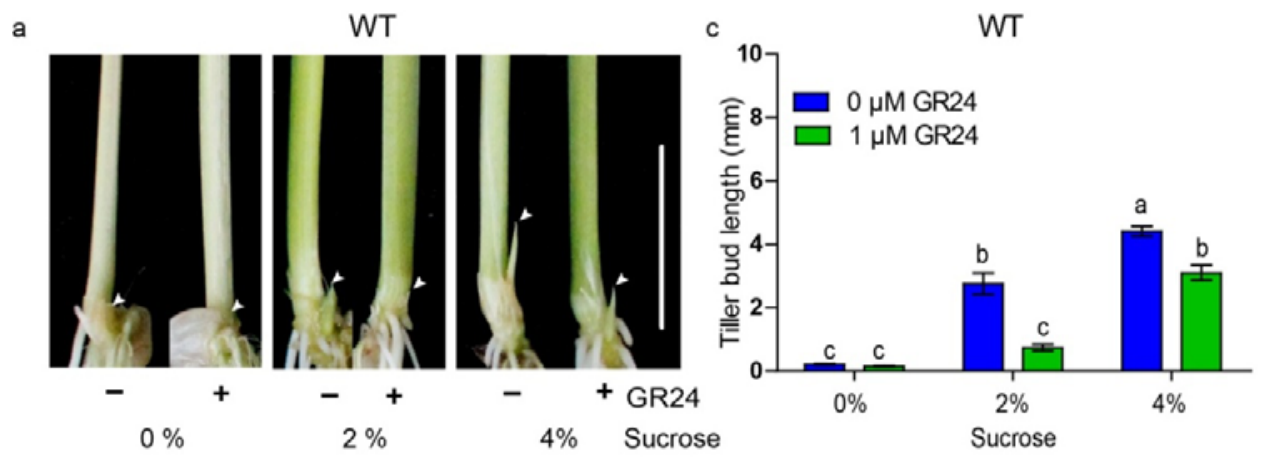

b

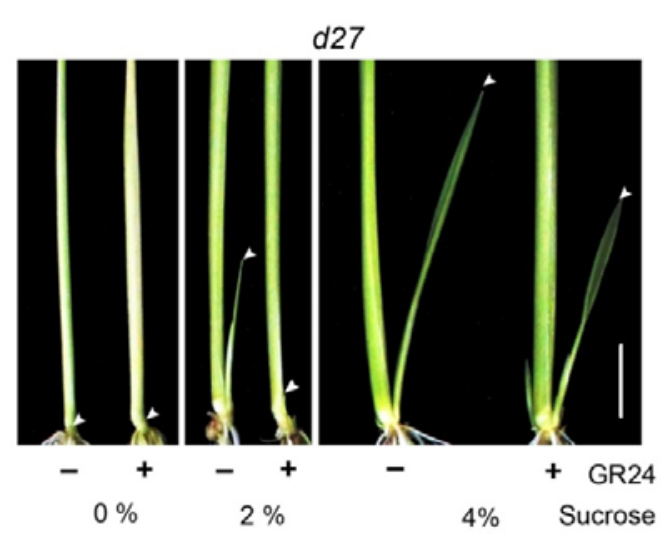

d
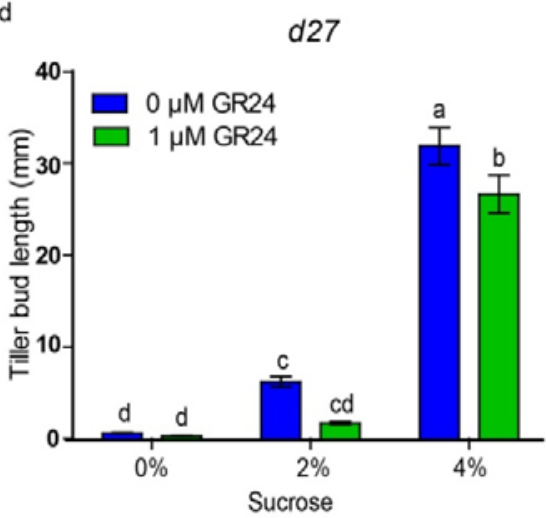

Figure 1. Sucrose alleviates the inhibitory effect of GR24 on tiller bud outgrowth in rice.

(a) Representative tiller buds of the WT (Huaidao5) and (b) SL-deficient mutant $d 27$, grown under different sucrose concentrations with or without $1 \mu \mathrm{M}$ GR24 for 3 weeks. Arrowhead represents tiller bud used for measurement. Scale bar represents $10 \mathrm{~mm}$. (c) Length of tiller buds of WT (Huaidao5) and (d) SL-deficient mutant d27grown on different sucrose concentrations with or without $1 \mu \mathrm{M}$ GR24 for 3 weeks. Different lower case letters denote significant differences $(p<0.05$, one-way ANOVA following Tukey's test for multiple comparisons). Error bars represent $\pm \operatorname{SE}(n>8)$. 
a

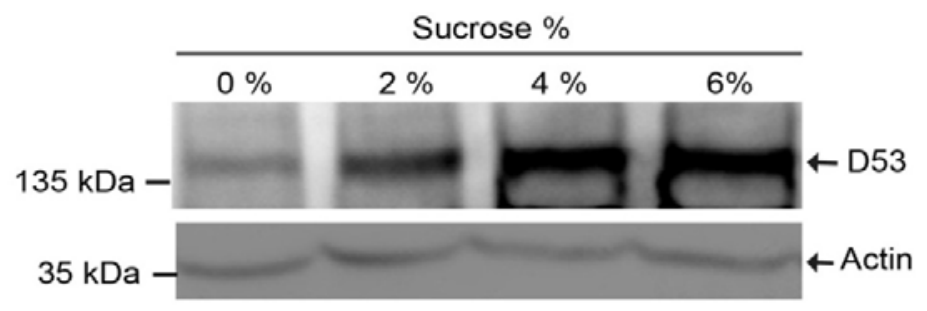

b

$0.5 \mu \mathrm{M}$ GR24

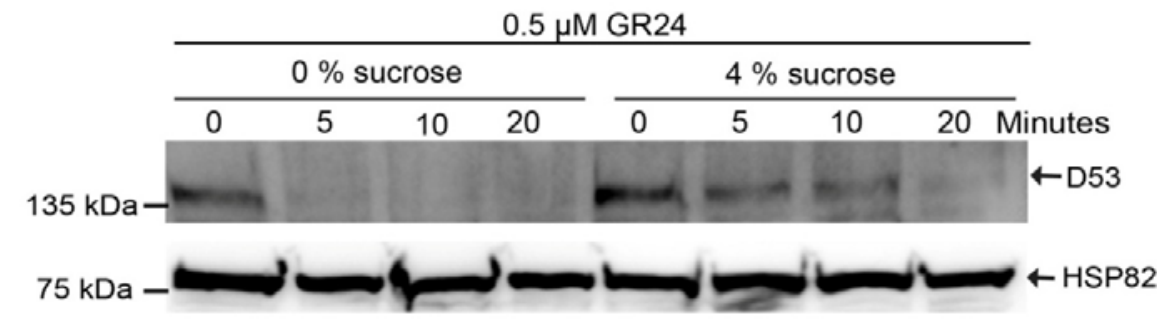

C

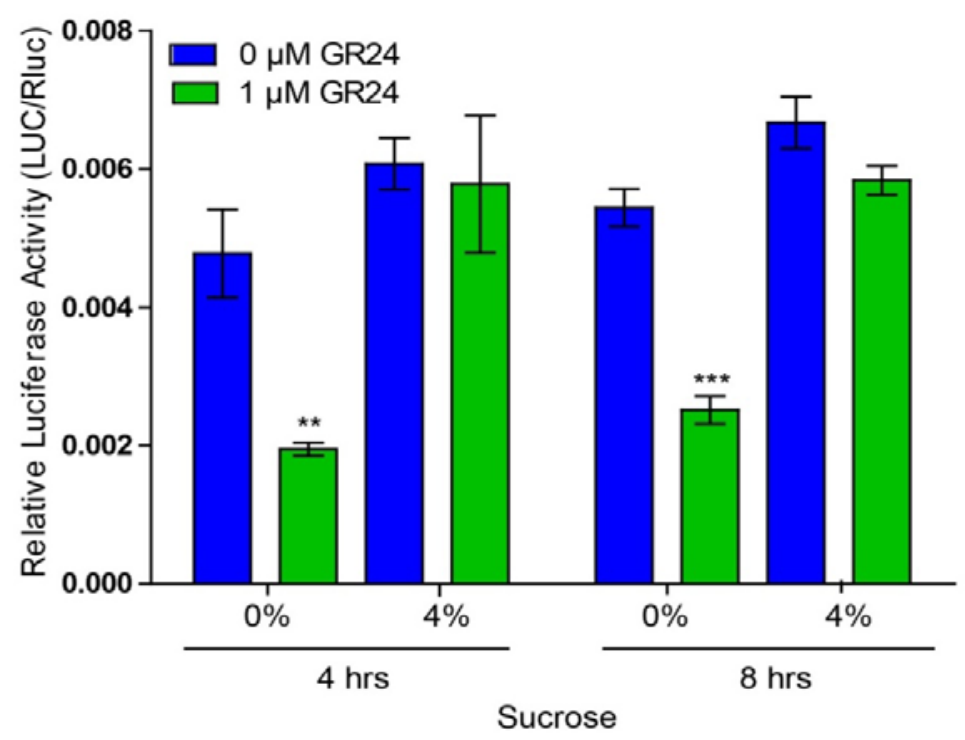

Figure 2. Sucrose alleviates the GR24-induced D53 degradation. (a) Western blot showing D53 protein accumulation in rice calli grown for 4 weeks on different sucrose concentrations. (b) D53 protein degradation in the WT rice calli initially grown on for NB plates containing $4 \%$ sucrose and later shifted to liquid media with or without $4 \%$ sucrose containing $0.5 \mu \mathrm{M}$ GR24 for different time points. (c) Transiently expressed luciferase-D53 protein in tobacco leaves subjected to $1 \mu$ M GR24 treatment with or without $4 \%$ sucrose for different time points. Luciferase readings were normalised with Renilla luciferase readings. Values are mean \pm SE $(n=4)$. Significant levels: $* * * p<0.001 ; * * p<0.01$; indicated by Student's t-Test. 


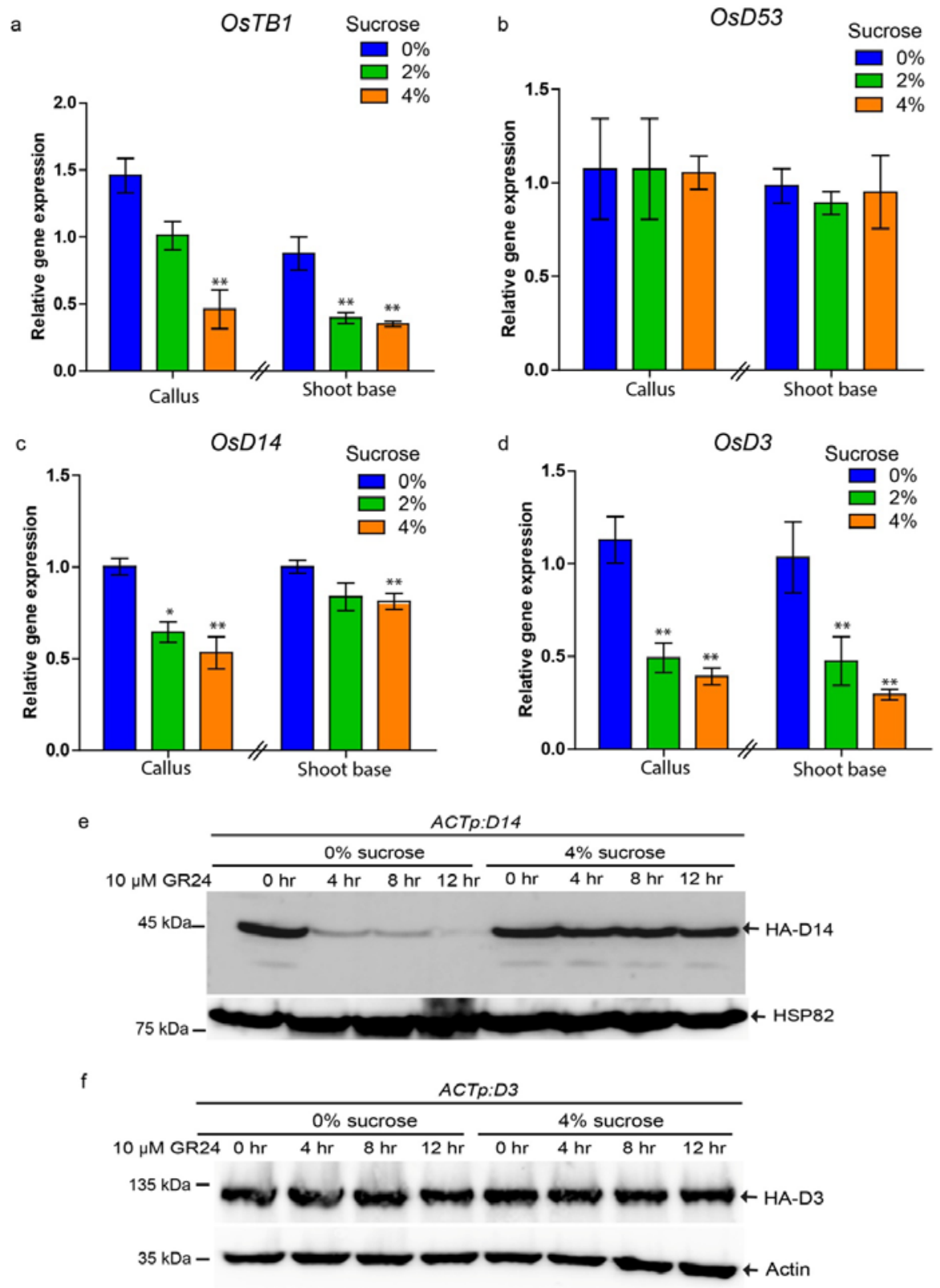

Figure 3. Sucrose down-regulates key genes in the SL signalling pathway. (a) Effect of different sucrose concentrations on the expression of TB1, (b) D53, (c) D14 and (d)D3 in the callus (grown for three weeks) and shoot base tissues of hydroponically grown rice seedlings (without sucrose) and treated with sucrose for $24 \mathrm{~h}$. Values are mean \pm SE $(n=3)$. Each replicate consists of 8 biologically independent samples. Significant levels: $* p<0.05, * * p<$ 0.01; indicated by Student's t-Test. (e) Effect of sucrose on GR24-mediated degradation of HA-tagged D14 fused protein levels detected by immunoblotting with an anti-HA tag monoclonal antibody. (f) Effect of sucrose on GR24-mediated degradation of HA-tagged D3 fused protein levels detected by immunoblotting with an anti-HA tag monoclonal antibody. 
a

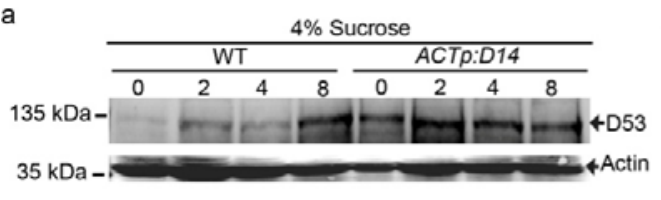

c

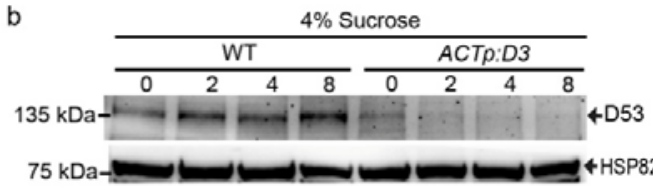

d

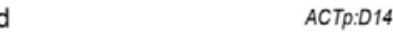

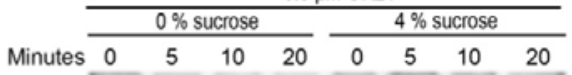

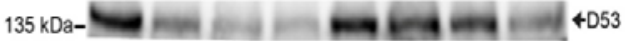
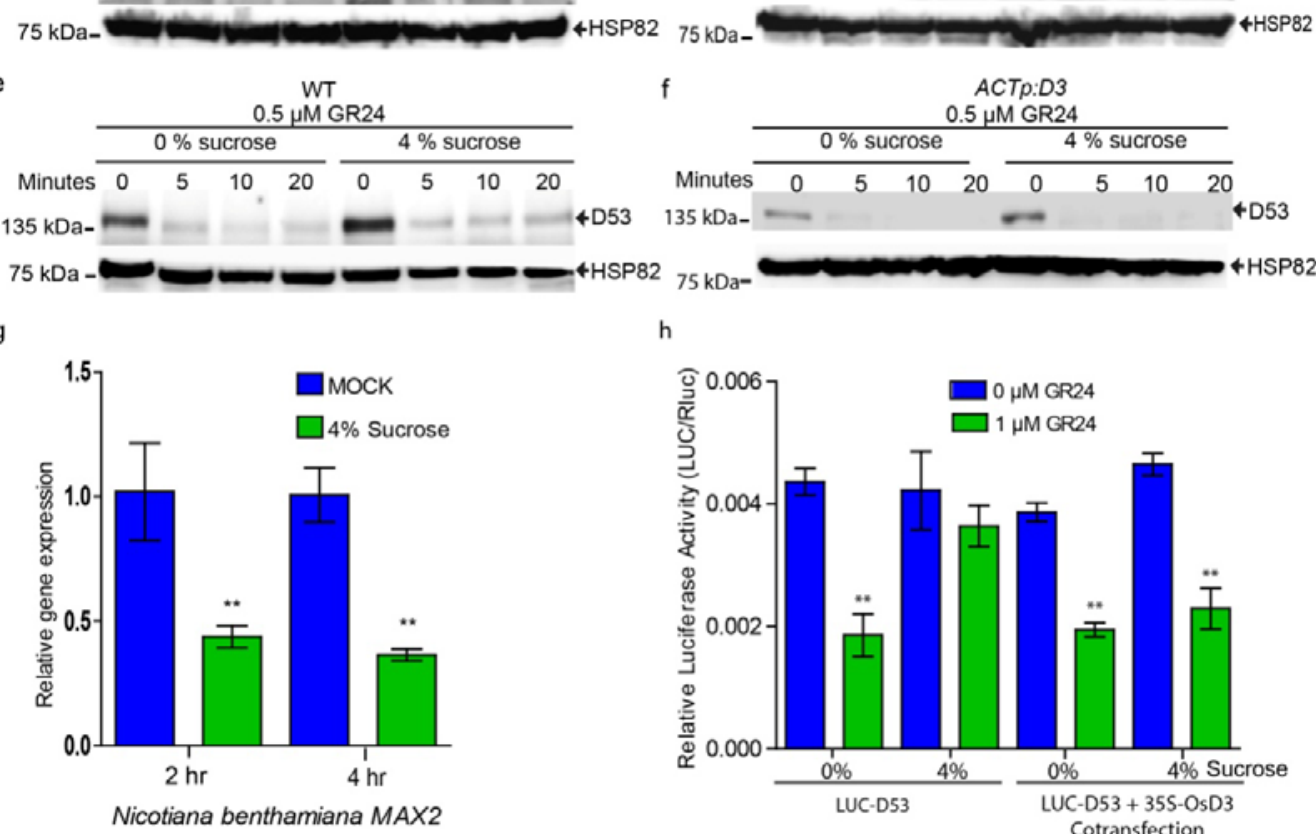

$\mathrm{h}$
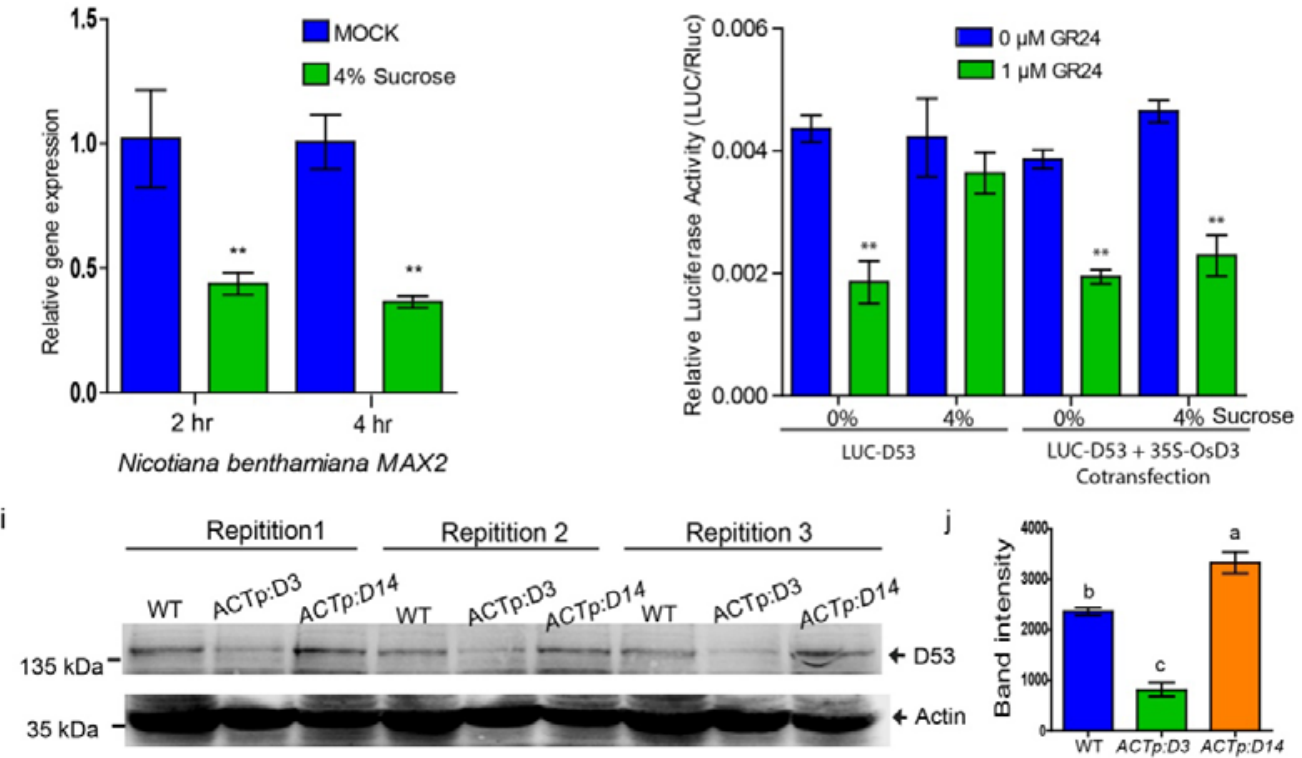

Figure 4. Over-expression of $D 3$, but not $D 14$, leads to D53 degradation in the presence of sucrose. (a) Effect of sucrose (4\%) on D53 accumulation at different time points in WT and D14 over-expression line (b) WT and D3 over-expression line detected by immunoblotting with an anti-D53 polyclonal antibody. (c) Effect of sucrose on D53 degradation in the calli of WT (GSOR300192), (d) D14 over expression line (e) WT (GSOR300002) and (f) D3 over expression line initially grown on NB plates containing $4 \%$ sucrose and later shifted to liquid media with or without $4 \%$ sucrose containing $0.5 \mu \mathrm{M}$ GR24 for different time points detected by immunoblotting with an anti-D53 polyclonal antibody (g) Effect of sucrose on Nicotiana benthamiana MAX2 (Nb MAX2) expression in tobacco leaves infiltrated with or 
without sucrose solution (4\%) at different time points. Values are mean \pm SE $(n=3)$. (h) Transiently expressed luciferase-D53 protein alone and co-transfected with 35S-OsD3 protein in tobacco leaves subjected to $1 \mu \mathrm{M}$ GR24 treatment with or without $4 \%$ sucrose for different time points. Luciferase readings were normalised with renilla luciferase readings. Values are mean \pm SE $(n=3)$. Significant levels: ${ }^{*} p<0.01$; indicated by Student's $t$-Test. (i) D53 protein levels in WT, ACTp:D3 and ACTp:D14 over-expressing lines grown on 4\% sucrose plates detected by immunoblotting with an anti-D53 polyclonal antibody. (j) Average band intensity of the D53 proteins quantified in the WT, ACTp:D3 and ACTp:D14 over-expressing lines using Image J software. Values are mean $\pm S E(n=3)$, Different lower case letters denote significant differences, $\mathrm{p}<0.05$, one-way ANOVA following Tukey's test for multiple comparisons. 


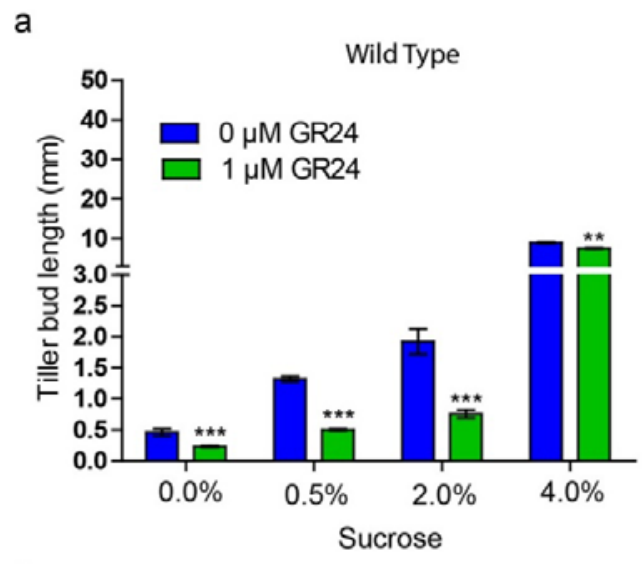

C

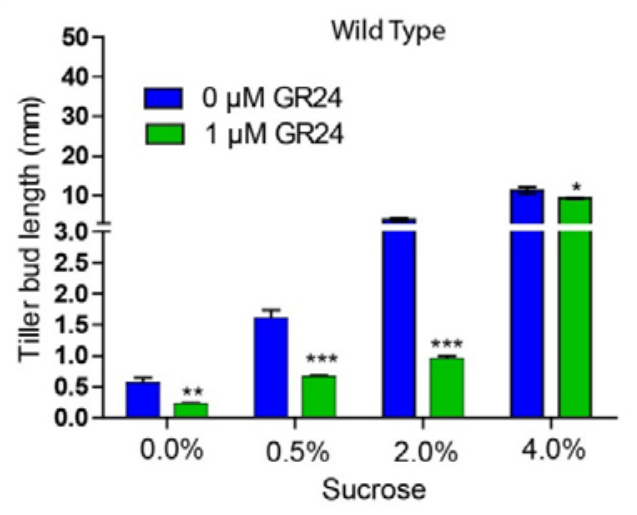

b

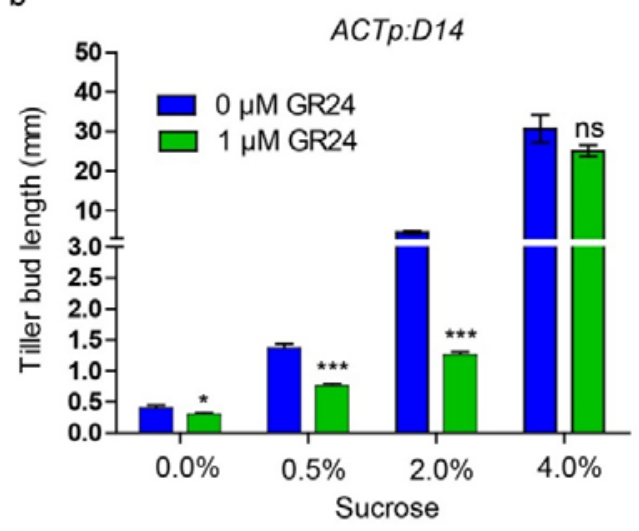

d

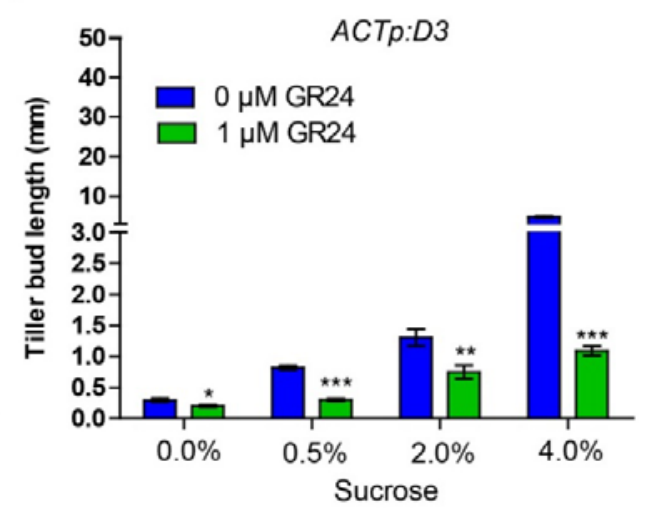

Figure 5. D3 over-expression prevents sucrose from inhibiting the SL-induced tillering suppression. (a) Effect of sucrose on tiller bud elongation in the WT (GSOR300192), (b) D14 over-expression line, (c) WT (GSOR300002) and (d) D3 over-expression line grown with or without $1 \mu M$ GR24 for 3 weeks. Values are mean \pm SE $(n=10)$. Significant levels: $* * * p<$ $0.001 ; * p<0.01, * p<0.05$; indicated by Student's $t$-Test. 
a

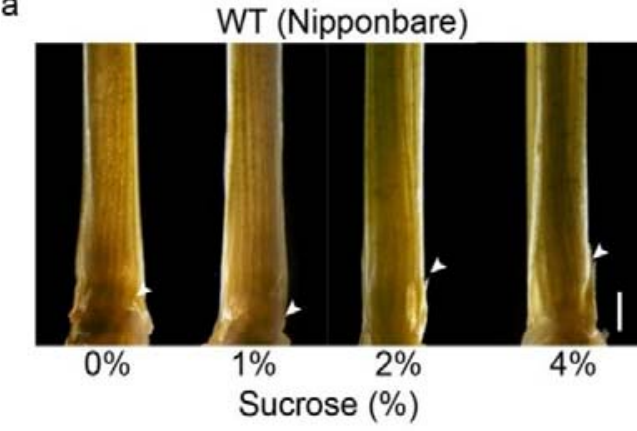

C

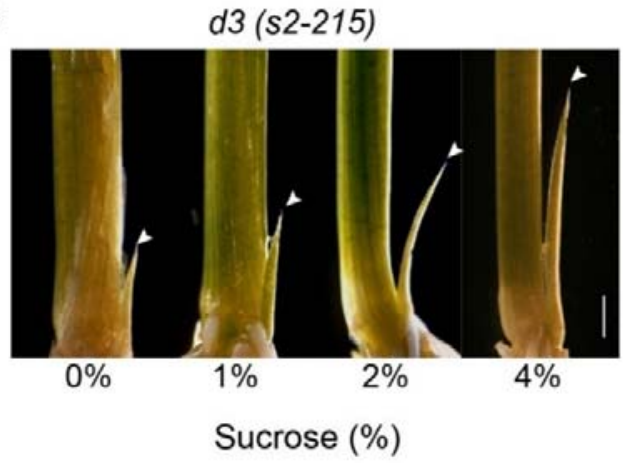

b
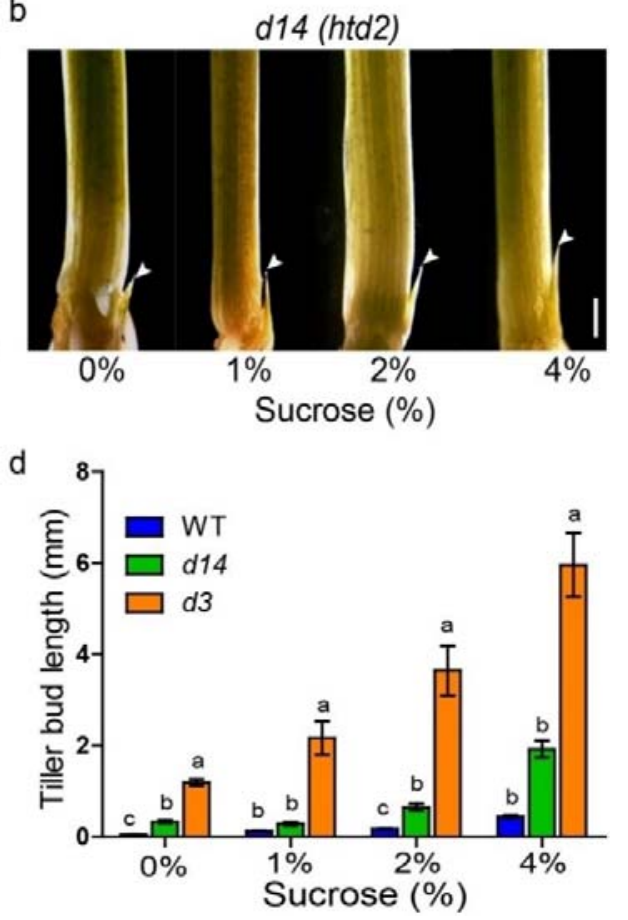

Figure 6. Disruption of $D 3$ increases the responsiveness to sucrose. (a) Representative images of tiller buds of WT (Nipponbare), (b) SL signalling mutants $d 14$ (htd-2) and (c) d3 (S2-215) grown on different sucrose concentrations for three weeks. (Scale bar= $1 \mathrm{~mm}$ ). Arrowhead represents tiller bud used for measurement. (d) Tiller bud outgrowth $(\mathrm{mm})$ in the WT (Nipponbare), $d 14$ (htd-2) and $d 3$ (s2-215) grown under the different sucrose concentrations for three weeks. Different lower case letters denote significant differences, $p<0.05$, one-way ANOVA following Tukey's test for multiple comparisons (compared for each sucrose concentration separately). Error bars represent \pm SE $(n>8)$. 

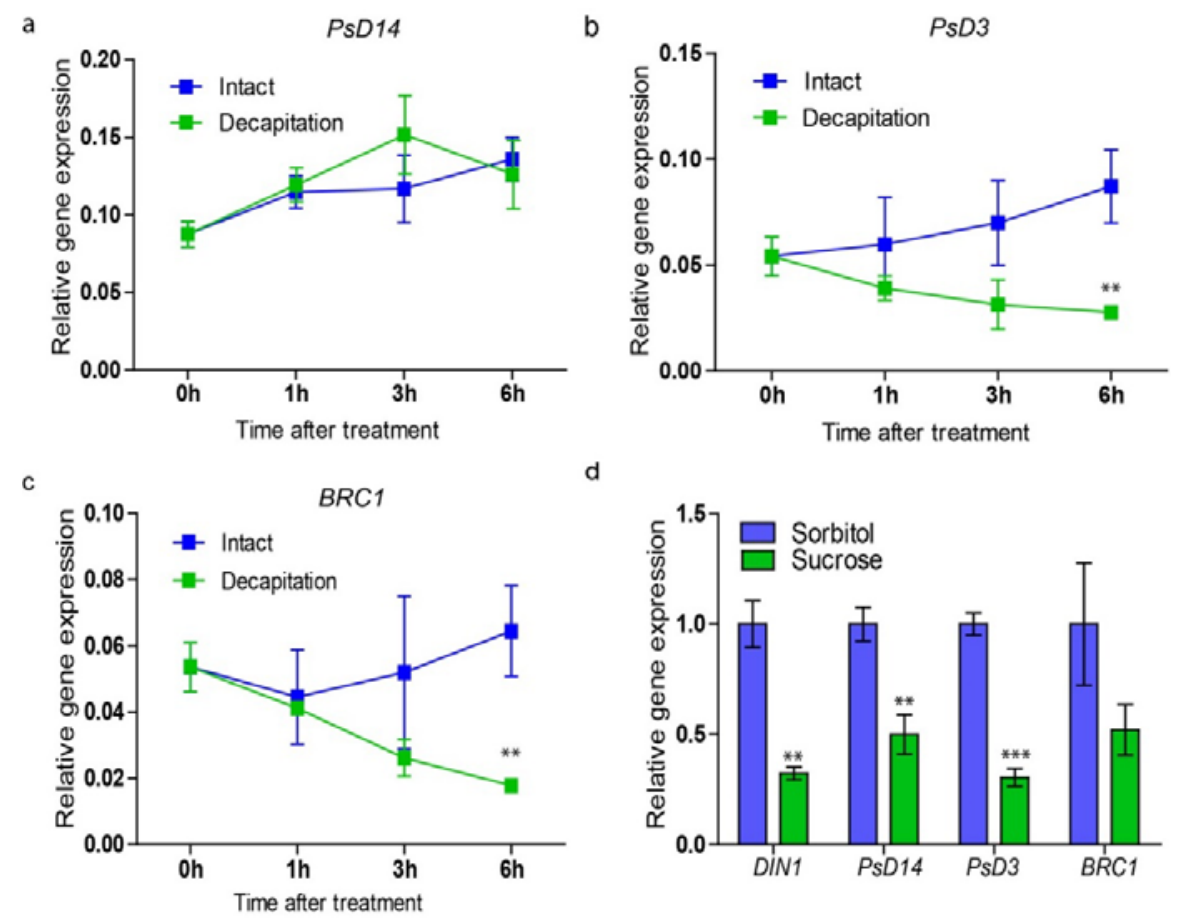

d

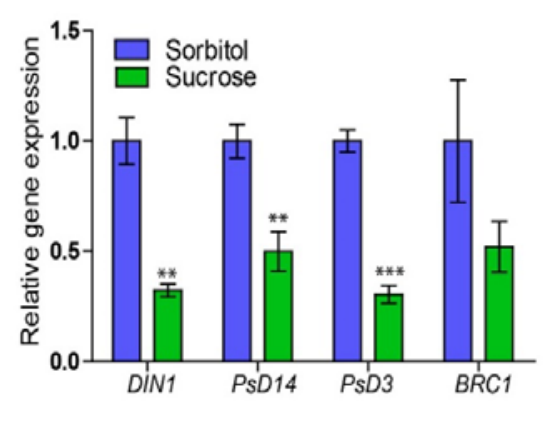

e

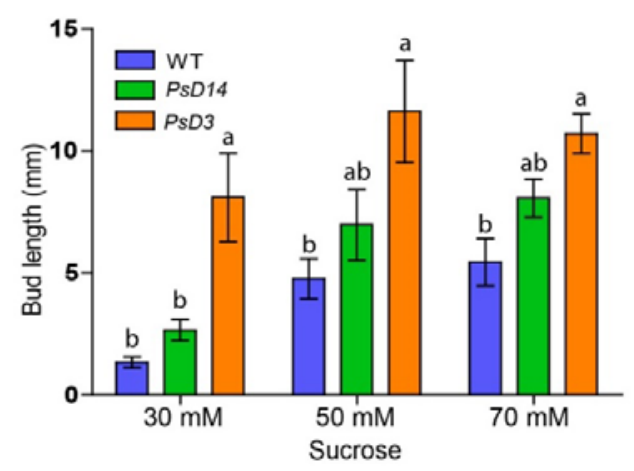

Figure 7. Effect of sucrose and decapitation on bud elongation and expression of SL pathway genes in pea buds. (a) Effect of decapitation on gene expression of $P S D 14$, (b) PsD3 (RMS4) and (c) BRC1 at different time points in response to decapitation. Values are mean \pm SE ( $n=3$ pools of 20 buds). (d) Expression of SL signalling genes in axillary buds fed with sucrose or sorbitol (osmotic control) through the petiole for $4 \mathrm{~h}$. Values are mean $\pm S E(n=3$ pools of 20 buds). Each replicate consists of 8 individual samples. Significant levels: $* * * p<$ $0.001 ; *{ }^{*} p 0.01$ indicated by Student's $t$-Test. (e) Length of single node pea buds of WT (Terese), rms3 and rms4 mutants grown in vitro with 30, 50 and $70 \mathrm{mM}$ sucrose for 6 days. Different lower case letters denote significant differences at each concentration $(p<0.05$, one-way ANOVA following Tukey's test for multiple comparisons). Error bars represent $\pm \mathrm{SE}$ ( $n=8$ individual buds). 


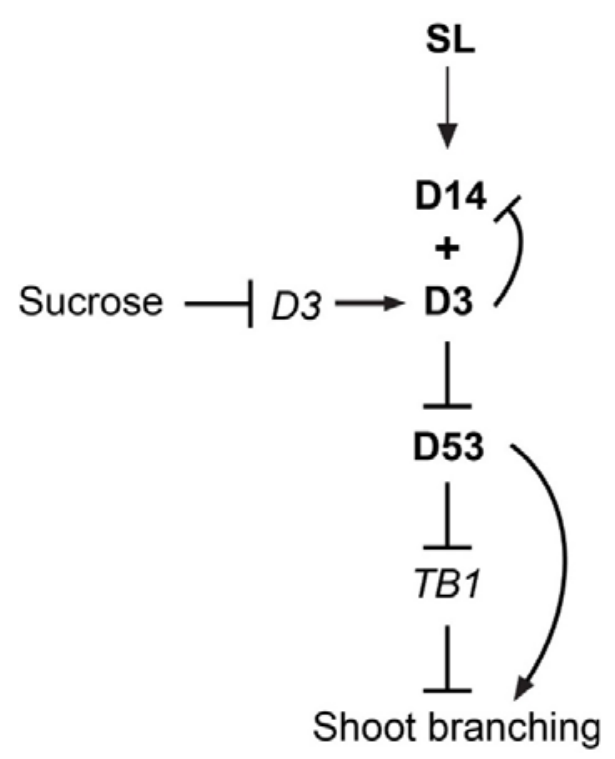

Figure 8. A proposed model of the interaction between sucrose and SL pathway. In the presence of sucrose, D3 transcript is repressed leading to a decrease in SL signal transduction. This ultimately stabilises the SL repressor D53 protein, which in turn triggers shoot branching, partly through TB1 inhibition. 